Carneiro, J.P., and Becerik-Gerber B.. (2017). "Impact of Immersive and Interactive Information Visualization on Occupant's Lighting Choices" In: LC3 2017: Volume I-Proceedings of the Joint Conference on Computing in Construction (JC3), July 4-7, 2017, Heraklion, Greece, pp. 465-473. DOI: https://doi.org/10.24928/JC3-2017/0210.

\title{
IMPACT OF IMMERSIVE AND INTERACTIVE INFORMATION VISUALIZATION ON OCCUPANT'S LIGHTING CHOICES
}

\author{
Joao P. Carneiro ${ }^{1}$ and Burcin Becerik-Gerber ${ }^{2}$
}

\begin{abstract}
Feedback systems often use Information Visualization (InfoVis) to inform occupants about the impact of their choices on energy consumption, aiming at reducing occupant behaviour related waste in buildings. In this pilot study, we use InfoVis as a tool to influence occupants' opinions about a room's lighting quality instead of their energy consumption. Lighting significantly affects occupants' mood, productivity, and wellbeing. We utilize InfoVis to present the light quality data throughout a day via an animated daylight cycle, as well as light levels and light distributions through static and animated heat maps to enable occupants to make more informed decisions related to lighting. InfoVis is presented within the spatial context by overlaying visualizations on the room itself, on its floor and the desk surface, using an immersive virtual environment. The results show that the participants changed their choices once provided with more information related to the quality and amount of lighting in the room. Specifically, the participants changed their choices significantly more when the illuminance levels and distribution over time (dynamic heat maps) were presented. The findings of this research can inform the development of better visualization techniques that could increase occupant awareness about environmental conditions in buildings.
\end{abstract}

Keywords: Immersive Virtual Environment, Immersive Interactive Visualization, Informed Decision, Lighting Data.

\section{INTRODUCTION}

Indoor environmental conditions in a building affect occupant mood, performance, satisfaction, and health (Veitch and Newsham 1998). Occupants are often more satisfied with a building if the building has personal control options (e.g., for thermostats, shading and lighting systems), which allow the users to set the indoor environment to their preferences (Deuble and de Dear 2012). However, the relationship between occupants and their use of personal controls is complex. Many variables influence the use of personal controls in buildings, such as user preferences and knowledge of the availability and accessibility of control options (Brown and Cole 2009). Occupants usually change their environment, or commit to a new choice when they are in an uncomfortable scenario. In most cases, occupants do not think about the consequences of their choices in advance but they usually focus on the easiest and quickest solution available (Leaman 1999).

To improve occupant choices toward pro-environmental behaviours, feedback systems that integrate some sort of Information Visualization (InfoVis) are used. Card et al. (1999) define InfoVis as "the use of computer-supported, interactive, visual representations of

1 Ph.D. Student, Sonny Astani Department of Civil and Environmental Engineering, University of Southern California, California, USA, jcarneir@usc.edu

2 Associate Professor, Sonny Astani Department of Civil and Environmental Engineering, University of Southern California, California, USA, becerik@usc.edu 
abstract data to amplify cognition." InfoVis has the potential to reach not only to the data experts, but also the novice users. Thus, InfoVis is becoming more present in our everyday life in assisting common tasks, such as home budgeting and tracking personal finances. Although users' choices in buildings could be based on many factors, such as feasibility, distance, availability and attractiveness of different options (e.g., view of a window, furniture, personal preference) (Cohen et al. 2008), the literature supports that InfoVis can also impact user behaviours in buildings.

We focus on understanding how InfoVis can be used to encourage users to make more informed decisions about environmental quality of a built environment. Specifically, we test how interactive and immersive visualizations impact occupant choices with regard to the quality of light in a room. Instead of focusing solely on energy consumption related visualizations, we present non-energy consumption data, focusing on daylight quality as well as light intensity and light distribution within a spatial context, which potentially improves the understanding of the presented data. To do so, we developed static and dynamic (animated) lighting related visualizations and presented them to the participants within a spatial context by using an immersive virtual environment (IVE). The spatial context (a single occupancy office) is presented in a 1:1 scale, including the office furniture, appliances and equipment. The proposed visualizations allow the user to visualize and interact with the data for different lighting choices before making any decisions regarding the room's light setting. Specifically, we presented animated daylight cycle where the participants can observe the room's lighting within a day and light levels/distributions in the room through static and animated heat maps and measured the impact of these visualizations on users' lighting related choices.

\section{BACKGROUND}

Research shows that occupants are willing to accept a certain level of discomfort if they are more conscientious of environmental issues (Deuble and de Dear 2012). Additionally, the knowledge of how a building will perform when a certain control choice is made directly affects occupant choices (Brown and Cole 2009). A literature review by Galasiu and Veitch (2006) shows how occupant preferences change based on many factors, such as daylight availability, visual comfort and glare, and window orientation and size, effecting the space's lighting quality. In majority of the cases, a user acts only when the discomfort has reached an unacceptable level. For example, a research study analysed the occupants' coping mechanisms for discomfort situations, and showed that certain coping mechanisms, such as the ones caused by glare on the computer screen, could be harmful for the occupants' wellbeing (Heerwagen 1992).

To reduce occupant coping mechanisms, interactive communication and feedback systems are used to increase occupant knowledge about their actions or choices in the building. These tools have been extensively studied in literature. For example, ecovisualization is a communication tool that is commonly studied and developed to impact occupant behaviours towards a more energy efficient behaviour. These visualizations have focused on showing energy consumption data to users in real-time and interactive fashion (Holmes 2007). Pierce et al. (2008) divides eco-visualizations based on their intended strategy, which varies with the characteristics of the data (i.e., granularity or number of disaggregated appliances), building type (i.e., residential or commercial) and users' motivations to save energy. However, these systems often focus on energy data rather than building performance data, which could lead occupants to more informed decisions 
about a building system and potentially convince users to choose more comfortable and perhaps sustainable choices.

With the recent advancements on virtual reality (specifically immersive virtual environments) and augmented reality systems, we now have the possibility to visualize building abstracted data (e.g., lux level, airflow, air temperature and etc.) in the spatial context of a building. The immersive characteristics of these tools allow the user to better understand the provided visualization by extending their perception (Taylor et al. 1996). In addition to extending user perception, IVEs also allow researchers to better isolate the studied variables (Jerald 2015). In this study, we focus on providing occupants the information about the effect of their choices on the built environment by providing immersive and interactive visualizations, using IVEs and enabling them to make more informed decisions about lighting in a built environment. Due to the relationship between occupants' choices and their resulting comfort, comfort-related data (i.e. illuminance distribution and daylight cycle) are displayed in the form of visualizations. Additionally, we use IVE's spatial capabilities to improve comprehension of the visualization by helping users associate their choices with their corresponding impacts on the built environment.

\section{METHODOLOGY}

The objective of this pilot study is to understand the impact of immersive and interactive InfoVis on the participants' lighting choices. We have selected two lighting related information as the basis for our visualizations: (1) illuminance levels, luminous per unit area (represented in lux) and (2) time (represented through a daylight cycle). This information is presented to the participants separately or in a combined fashion, which resulted in three visualization types: (a) a static light map (lux levels), (b) daylight cycle (time), and (c) animated light maps (lux levels and time). The visualizations use two techniques to display the information to the participants, colour maps (or heat maps), and data animation. Colour maps are used to demonstrate the light distribution and intensity at each point in the room. Specifically, we used the room illuminance levels (lux levels) to generate the colour maps. The data animation is used to emulate time. The animation is applied to generate the daylight cycle, in other words, the natural light in the room changes based on the building's location/orientation and time. The lux level data are dependent on time and the environment's geometry, which creates a dependency between the environment and the presented data. This dependency improves a participant's understanding of the room's lighting quality. In our studies, we have selected a daylight cycle animation for a building located in Los Angeles, California, on December 22nd of 2016, from 9 am to $5 \mathrm{pm}$.

Based on the InfoVis techniques used in this study, we developed four environments to measure the impact of visualizations on the participants' lighting related choices: the control environment (environment 0) and the InfoVis environments (environments 1, 2, and 3). In environment 0 (Figure 1), the room's lighting was visualized at two static times (i.e., 9am and 1pm). Environment 1 was similar to the environment 0 , with the addition of a static heat map, showing the lux levels/distributions overlaid on the floor and desk for $9 \mathrm{am}$ and 1pm (Figure 1). Environments 2 and 3 had two time zones, morning (9 am to 1 $\mathrm{pm})$ and afternoon ( $1 \mathrm{pm}$ to $5 \mathrm{pm})$. We selected $1 \mathrm{pm}$ as the breaking point between the morning and the afternoon because at $1 \mathrm{pm}$, the natural light would not produce glare on the computer screen and it also generates two equal periods of 4 hours in a regular office workday (9 am to $5 \mathrm{pm}$ ). We used two time zones to understand if the participants' choices were impacted by the presented InfoVis or their personal preferences due to the amount 
of available lighting in the room. Environment 2 presented an animated dynamic daylight cycle for the two time zones, each one took 1 minute (see https://www.youtube.com/watch?v=WmcsYIA0kBY). Finally, Environment 3 presented a dynamic (the daylight cycle) animated heat map over layered on the floor and desk for the two time zones (see https://www.youtube.com/watch?v=zG5FdrDr js).
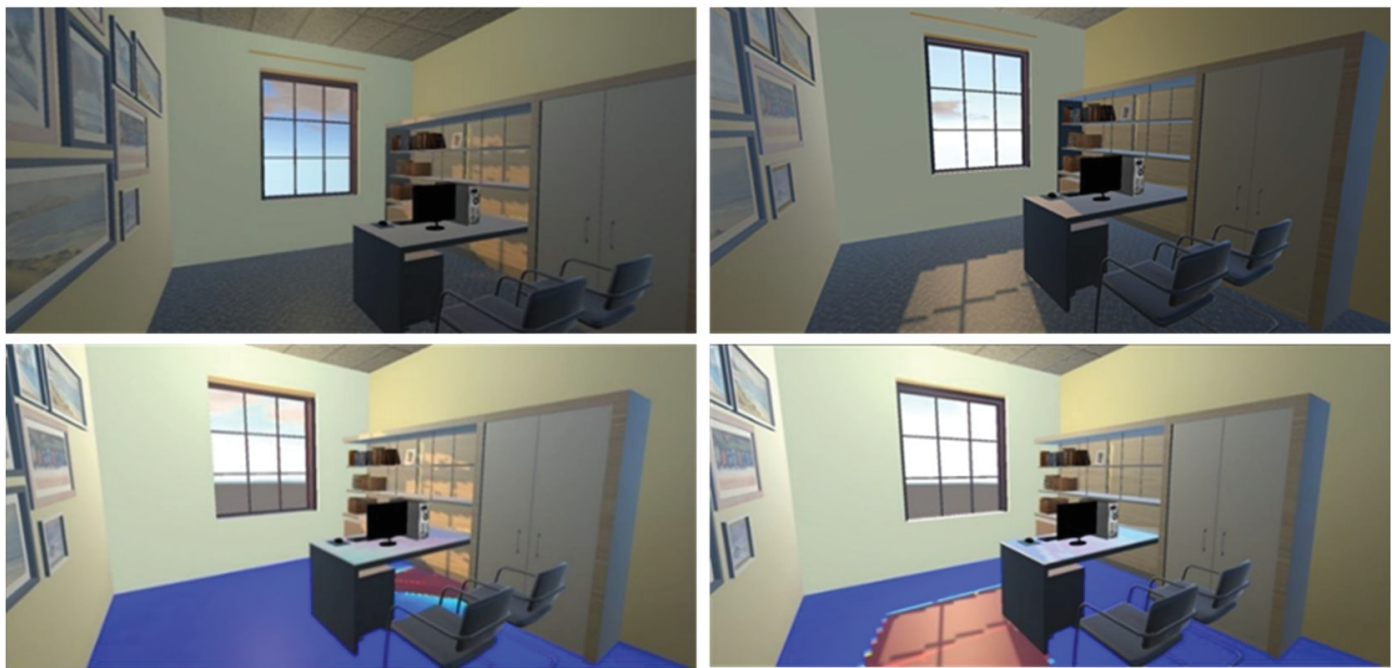

Figure 1: ENV 0 at 9am (top left); ENV 0 at 1pm (top right); ENV 1 at 9am (bottom left); ENV 1 at $1 \mathrm{pm}$ (bottom right)

Modelling: The first step of the workflow was to create the studied environment -- a single occupancy office located in Los Angeles. The office has 17 square meters (3.65m by $4.65 \mathrm{~m}$ ) of floor area. The room has one window and one lighting fixture. The office environment was designed in Revit ${ }^{\circledR}$ 2016, where the basic geometry (e.g., walls, ceiling, floor, door, and window) and the basic room furniture (e.g., cabinets, book shelves, office desk, chairs) were added to the environment. After, the model was taken to 3ds Max ${ }^{\circledR}$ to improve the geometry and fix the UV maps (materials).

Lighting Simulation and Light Maps: The second step was to simulate the light intensity data (room lux levels) and to generate the heat maps. The light simulations were executed in Rhinoceros $5{ }^{\circledR}$ with the assistance of plugins: Grasshopper ${ }^{\circledR}$, Honeybee ${ }^{\circledR}$ and Ladybug ${ }^{\circledR}$. The environment had two analysed areas: the room's floor, and the surface of the office desk. After the simulations were completed, the data were exported to Matlab® to generate the heat maps. 160 animated heat maps were generated (one map for every 3 minutes) and were then compressed into an animation.

Immersive Virtual Environment: The third step was the development of the interactions and the final versions of the models in Unity ${ }^{\circledR}$, which was used to create the immersive interactive environments with a head mounted display (i.e., Oculus Rift $\left.{ }^{\circledR}\right)$. The interactions added to the environments were opening and closing of the blinds and turning the lights on/off. Materials were added to increase scene realism by adding different texture maps based on the objects UV maps, which were generated in the previous step. These maps were: normal, specular, occlusion, and displacement maps, which all influence the objects reflections and global illumination. Finally, High Dynamic Range Rendering (HDRR) and tone mapping were used to improve the environment's light realism by expanding the camera colour range.

Experiment Design: At the beginning of the experiment, the participants were asked to review a consent form approved by the IRB. If the participants agreed to take part in the 
experiment, they were asked to fill out a pre-experiment survey. Below is the information collected in this survey:

- Population demographics - This information was collected to identify the population age, education level, and gender.

- Vision problems - Before we run any experiment, we ensured that participants can properly visualize the models and the information (for example, colour blindness). Depending on the participant's answers to this set of questions, he/she was qualified or not qualified to participate in the study.

If the participants were qualified to take part in the study, we placed them in an immersive virtual training environment, which was a different office environment in a different location, time, furniture and lighting set up. Through the training, they were exposed to and became acquainted with the immersive interactions available in the environment. After the training phase, all participants started in environment 0 where the time was static (i.e., time did not vary within the experiment) and no visualization was present. They were randomly assigned to the morning time ( $9 \mathrm{am}$; time 1$)$ or to the afternoon time (1 pm; time 2). Whether on time 1 or 2, they were asked to get familiar with the room and set up the lighting based on their preferences (initial set up was a dark room). Participants had the options to open/close the blinds or turn on/off the lights. Afterwards, they were asked to watch a two-minute video, which was intended to distract the participants and reduce the carryover effects to the next stage of the experiment. At the end of the video, the participants were asked to repeat the procedure at another time (e.g., if initially assigned to 9am then they got assigned to $1 \mathrm{pm}$ and vice versa). Then, they completed the personality survey, explained below:

- Big five personality test - We measured the participant's personality, (John and Srivastava 1999), which helped us understand if the personality of the participant affected the choices or not.

Next, the participants were randomly assigned to one of the InfoVis environments (environments 1, 2, or 3), 10 participants in each environment. In their assigned environments, the participants were asked to interact with the lighting options to observe how the visualization changed in real time. Each participant was assigned to the same experimental procedure as explained above for the environment 0 . In each time zone, the participant was presented with the information related to that time. For example, if the participant was assigned to the environment 3 (animated light map), the map would follow the daylight cycle for each time interval. Once the participants interacted with different lighting options and have seen different visualizations in real time and made their final choices regarding lighting, we brought the environment to either 9am or 1pm and asked the participants to set up the lighting to their preferred levels. As the final step of the experiment, participants were asked to answer the post-environment survey to collect the following information:

- Sense of presence - The presence survey was developed by Witmer and Singer (1998). The sense of presence is crucial because the effectiveness of the virtual environments is often linked to the sense of presence. At the same time, the sense of presence enables the sense of immersion, encouraging user involvement.

- Understanding of the information - This survey was developed to analyse the participants' understanding of the presented InfoVis and the participants' desire to learn about lighting. 
- Lighting design knowledge - This survey was designed to measure participants' knowledge about lighting and lighting systems.

- Environmentalism- The survey was designed to measure the participants' environmental views and values.

Data Analysis: As the last step, we analysed a binary set of data that shows if the participants kept their choices (0) or changed their choices (1). The binary data provided the following information: a) if the participants have changed their choices between the two time zones in an environment, and b) if the participants changed their choices between environment 0 and the InfoVis environment, to which they were assigned (environment 1, 2 or 3). The comparisons were made between the same time zones. We analysed the data in two ways between the participants of the same group and between groups. For the in-group comparison, we used the change rate to understand if the lighting choices were impacted by the quality of light. After the in-group comparison, we compared the data in pairs to measure the impact of each InfoVis environment on the participants' choices. For example, we compared environment 1 with environment 2 . In order to measure if there is any significant difference between the groups and which InfoVis environment was more impactful, we have used Chi-square tests between the pairs.

\section{RESULTS}

For this pilot study, we collected 30 participants' data, 13 females and 17 males. The age range varied from 20 to 32 years with an average age of 25 and standard deviation of 3 years. $80 \%$ of the study population were students. None of the participants had any vision problems that would disqualify them from participating in the study.

In order to demonstrate the participants' choices, we have developed an interactive Sankey diagram, also available at http://joaopcarneiro.com/ConVRGraphs/alluvial.html). Each diagram has 3 columns (or nodes), which represent: 1 -participants' choices in the controlled environment (environment 0 ); 2 -participants' choices in the environment with visualization (environment 1,2, or 3); and 3 - if participants have changed their choices between the environments or maintained them the same. Additionally, the colour shows if the participants' choices increased or reduced the energy consumption between the two environments (control and InfoVis environments). The energy consumption was calculated using Rhino ${ }^{\circledR}$, Grasshopper ${ }^{\circledR}$, Honeybee ${ }^{\circledR}$ and Ladybug ${ }^{\circledR}$, following a similar approach used to calculate the office illuminance levels. These tools use well known physical simulation engines, such as EnergyPlus ${ }^{\circledR}$, Daysim ${ }^{\circledR}$, and Radiance ${ }^{\circledR}$ to calculate lighting related consumption, as well as the impact of heat gain on the office's cooling system. In order to have more realistic results, we created a full building geometry, with five floors, five offices per floor, and one hallway per floor. Even though we used a building model for the simulation, we only used the energy results of one centre office room located on the third floor. The energy simulation results are for average consumption of the studied day (December 22, 2016), including the heat gain resulting from the operation of blinds.

Based on the collected data, we found a change rate of $37 \%, 20 \%, 30 \%$ and $40 \%$ for environments $0,1,2$ and 3, respectively, between morning and afternoon. This shows a small variance between the morning and afternoon choices. Based on the Chi-square test, no statistical significance was found between the morning and afternoon, meaning that the changes were not affected by the difference in the availability of natural light. This shows that the participants' choices were not affected by the different preferences that 
they might have between morning and afternoon. The change rates between the morning without visualization (environment 0 ) and the morning with visualization were $30 \%, 70 \%$, and $80 \%$ for environment 1, 2, and 3, respectively (Figure 2 - top graphs). Based on the pair comparisons between the environments, there was a significant difference between environments 1 and 3. In addition, there was a marginally significant difference between environments 1 and 2. This shows that the participants reconsidered their choices once they were presented with dynamic visualizations. As for the afternoon, the change rates were $30 \%, 40 \%$, and $70 \%$ for environments 1, 2, and 3, respectively (Figure 2 - bottom graphs). In the Chi-square test of these results, we did not find any significant difference. However, there was a marginally significant difference between environments 1 and 3 . These results show the dynamic heat map (environment 3) to influence lighting related choices. This results are demonstrated clearly in figure 2, for example, the "true" column, which shows if a change has occurred or not, is larger in group 3 than any other group. It is important to note that the changes were not more or less energy efficient, meaning that once the change occurred it could either be more sustainable or not, as the figure 2 presents.

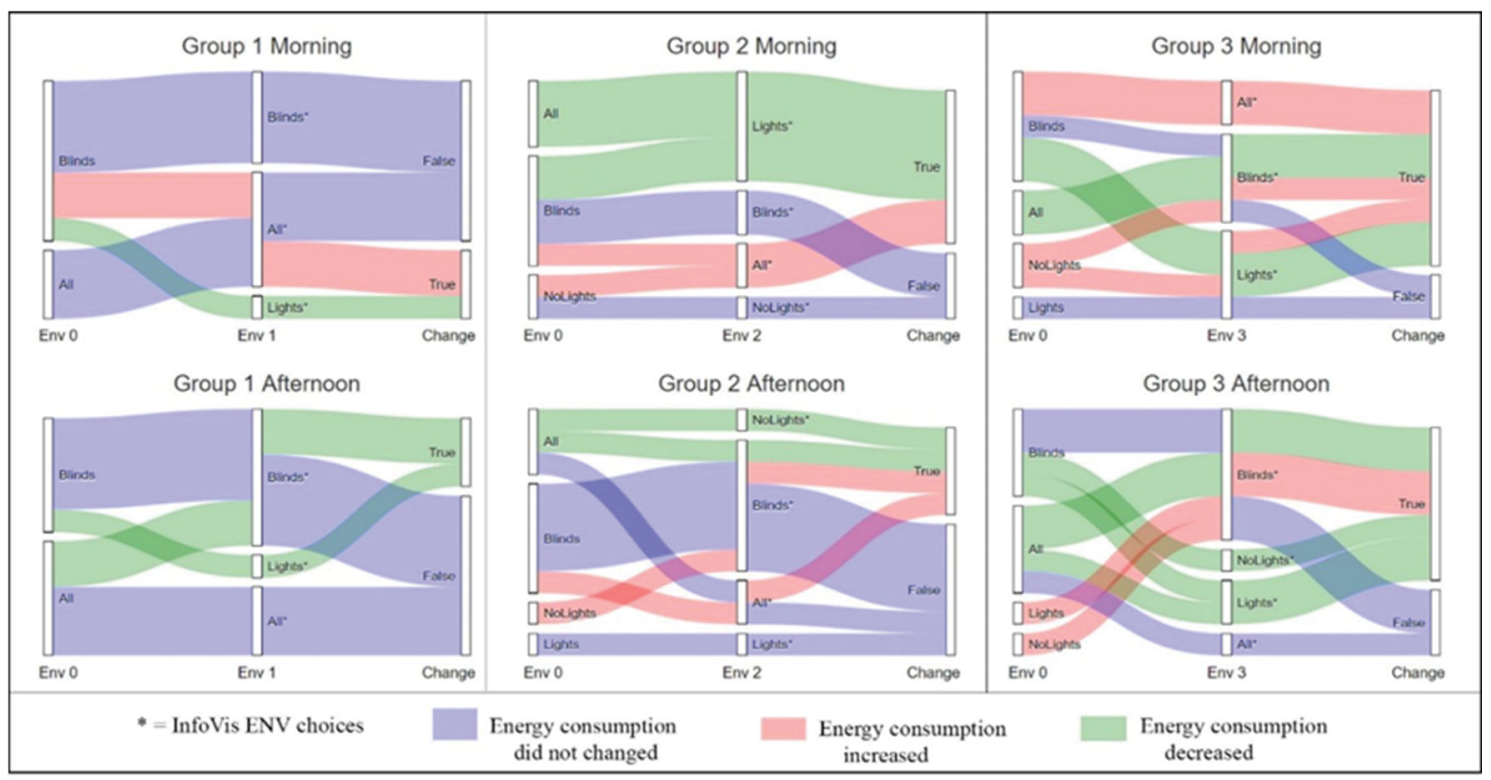

Figure 2: Sankey diagram of participants' choices

Based on the survey results, the participants easily understood the presented information and they believed that the information was adequate to make them reconsider their choices. $70 \%$ of the participants were extremely satisfied or satisfied with the presented visualizations, $63 \%$ found it easy to understand the visualizations and $77 \%$ thought there was adequate information for them to make their decisions. These results show that the designed information have met the purpose of conveying information to the participants. The presence survey showed that the participants had a satisfactory perception of the four measured factors (i.e., control, sensory, realism and distraction). For example, the two questions that allowed us to measure the realism received positive feedback from the participants, in that $63 \%$ answered that their experience in the virtual environment seemed to be consistent with the real world and $77 \%$ answered that they were able to actively survey the environment with their vision. Overall, the questionnaire results showed that most of the participants were fully engaged in the virtual environment, which makes the experiment results comparable to the real-world results. 
We did not find any significance between the personality traits and the participant choices or the change rates; however, this could be due to the small number of participants we had in each group. In the next phase of the study, we will examine if the personality traits had any influence on the final choices. In addition, the participants tend to prefer natural light, $80 \%$ of the participants either strongly agreed or somewhat agreed that they prefer natural light in the real world. Participants did not think that they had adequate knowledge about lighting or lighting systems, for example, $50 \%$ of the participants were neutral and $23.3 \%$ answered they did not know enough about lighting or lighting systems. Since their knowledge about lighting or lighting systems were not sufficient, we can presume that the presented InfoVis did impact their choices.

\section{CONCLUSION}

In this study, we analysed the impact of interactive and immersive information visualizations on occupants' choices. The results showed that the dynamic light map (environment 3) was the only visualization to have a significant effect on the user choices. Participants easily understood the presented information and considered it adequate enough to make decisions. At the same time, the participants' final choice was not necessarily more sustainable. In this study, our visualizations were mainly for conveying lighting related information rather than convincing people to make more energy efficient choices.

The study has some limitations. One limitation is the small sample size, which could have affected the results. Another limitation is the interaction options with the blinds. In our study, the blinds were set to be fully open or fully closed, and nothing in between. In order to address these limitations, we plan to improve the experiment design and run a full experiment with a larger study population. We will continue to explore how different immersive and interactive visualizations impact occupant choices by adding energy consumption visualizations and measure the impact of visualizations on energy consumption patterns. In our future studies, we plan to use augmented reality to address certain limitations come with IVEs and improve our experiments. These technologies will allow us to apply the visualizations presented in this paper to occupants' everyday life and conduct multi-day evaluations. Overall, this study has allowed us to begin to understand which visualization techniques can be efficient and are impactful means to present nonenergy consumption information to users in order to influence their choices to increase comfort while reducing unsustainable behaviour.

\section{REFERENCES}

Brown, Z. and Cole, J. (2009) Influence of occupants' knowledge on comfort expectations and behaviour. Building Research \& Information, 37, 227-245.

Cards, S.K., Mackinlay, J.D. and Sheiderman, B. (1999). Readings in information visualization: using vision to think, Morgan Kaufmann.

Cohen, J.B., Belyavsky, J. and Silk, T. (2008). Using visualization to alter the balance between desirability and feasibility during choice. Journal of Consumer Psychology, 18, 270-275.

Deuble, M.P. and De Dear, R.J. (2012). Green occupants for green buildings: the missing link? Building and Environment, 56, 21-27. 
Galasiu, A.D. and Veitch, J. A. (2006). Occupant preferences and satisfaction with the luminous environment and control systems in daylit offices: a literature review. Energy and Buildings, 38, 728-742.

Heerwagen, J. (1992). Adaptations and Coping: Occupant Response tn Discomfort in Energy Efficient Buildings.

Holmes, T.G. (2007). Eco-visualization: combining art and technology to reduce energy consumption. Proceedings of the 6th ACM SIGCHI conference on Creativity \& cognition, 2007. ACM, 153-162.

Jerald, J. (2015). The VR Book: Human-Centered Design for Virtual Reality, Morgan \& Claypool.

John, O.P. and Srivastava, S. (1999). The Big Five trait taxonomy: History, measurement, and theoretical perspectives. Handbook of personality: Theory and research, 2, 102138.

Leaman, A. (1999). Window seat or aisle. Architects' Journal, 3.

Pierce, J., Odom, W. and Blevis, E. (2008). Energy aware dwelling: a critical survey of interaction design for eco-visualizations. Proceedings of the 20th Australasian Conference on Computer-Human Interaction: Designing for Habitus and Habitat, 2008. ACM, 1-8.

Taylor, V.E., Stevens, R. and Canfield, T. (1996). Performance models of interactive, immersive visualization for scientific applications. High Performance Computing for Computer Graphics and Visualisation. Springer.

Veitch, J.A. and Newsham, G.R. (1998). Lighting quality and energy-efficiency effects on task performance, mood, health, satisfaction, and comfort. Journal of the Illuminating Engineering Society, 27, 107-129.

Witmer, B.G. and Singer, M.J. (1998). Measuring presence in virtual environments: A presence questionnaire. Presence: Teleoperators and virtual environments, 7, 225-240. 